\title{
Equilibrium Play in Voluntary Ultimatum Games: Beneficence Cannot Be Extorted *
}

\author{
Vernon L. Smith ${ }^{\dagger}$ and Bart J. Wilson ${ }^{\ddagger}$ \\ Economic Science Institute \\ Smith Institute for Political Economy and Philosophy \\ Chapman University \\ Orange, CA 92866
}

19 July 2017

\begin{abstract}
One robust result in experimental economics is the failure to observe equilibrium play in the ultimatum game. A heretofore unnoticed feature of the game is that neither player voluntarily chooses to play the game. Motivated by Adam Smith's proposition that beneficence-like that of nonequilibrium play in the ultimatum game-cannot be extorted by force, we offer the responder the opportunity to opt out of the game for a mere $\$ 1$ payoff for both players. We observe high rates of equilibrium play with highly unequal splits when responders choose to play such ultimatum games with both fixed and variable sums.
\end{abstract}

JEL Codes: C91

Keywords: ultimatum games, voluntary play, Adam Smith, experimental economics

\footnotetext{
${ }^{*}$ We thank Megan Luetje for recruiting our experiment participants, Jeffrey Kirchner for his professional software programming, and Chapman University for financial support to conduct the experiment.

+Email: vsmith@chapman.edu

‡Email: bartwilson@gmail.com
} 
Motivated by Adam Smith's propositions on beneficence in The Theory of Moral Sentiments (1759, pp 78-81; hereafter TMS), we study choice in new ultimatum games (UG) in which Player 1 can voluntarily signal a willingness to play the role of the Responder in a UG, and thereby enable play of the subgame. ${ }^{1}$ Using between-subjects treatments, we examine two new UG economic environments: (1) the standard UG for the division of a fixed sum, where the choice is between an equal split and an 11 to 1 equilibrium division-much higher than the 4 to 1 splits normally reported and in which, if offered, about half are rejected; (2) a variable-sum version in which Player 2 chooses between an equal split of a fixed sum and an investment option, with an equilibrium outcome that doubles the original stakes. In both games, based on a proposition in Smith $(1759, \mathrm{p} 78)$, we predict and find substantially increased support for equilibrium play relative to the received literature known to us. ${ }^{2}$ In the investment version we find that over three quarters of the Player 2's select the equilibrium option, and only 6 percent of the Player 1's veto the proposal. These data support the interpretation that the standard UG is played by what Daniel Ellsberg (1956) calls "reluctant duelists," a non-trivial condition relevant to predicting decisions, and embraced by Adam Smith's model of human sociability.

\section{The Ultimatum Game and Its Background}

In an ultimatum game the experimenter randomly assigns $2 N$ subjects to matched pairs, and in each pair one is randomly designated the Proposer the other the Responder. In early versions of the UG, a fixed sum, say $M$ dollars, is then divided by action of each of the paired players in a sequential

\footnotetext{
${ }^{1}$ In our experiment, an outside option allows self-selection to play or not a UG, whereas in the literature outside options have been used extensively to study expectations, offers and rejection rates by players while engaged in a UG that is required-play by the experimenter. This distinction illustrates how orthogonal the standard utilitarian model is to TMS, which is refreshingly new and predictively rich in empirical content. So far as we are able to determine none of the literature in experimental psychology or economics was inspired by Adam Smith. But see the early treatment by Ortmann and Meardon (1995).

${ }^{2}$ However, equilibrium play has been observed in "primitive" societies: "In some of these cultures, people did not think that sharing fairly was necessary... and Responders accepted nearly every offer. Ironically, these simple societies are the only known populations who behave exactly as game theory predicts!" (Camerer, 2003, p 11). Smith's model of human sociability seems to be consistent with these findings: "If our own misery pinches us very severely, we have no leisure to attend to that of our neighbor" (Smith, 1759, p 205).
} 
extensive form (or simultaneous-play, strategic form) procedure. The Proposer offers a division of the $M$ dollars in which the Proposer receives $M-x$, and the Responder receives $x$, where $0 \leq x \leq M$; the Responder then can accept this division yielding the proposed payments, or can veto the proposal, in which case each receives a payment of zero. The equilibrium of the game is $M-1$ dollars for the Proposer, \$1 (the minimum unit of account) for the Responder, with the Responder accepting the proposal because it strictly dominates rejection.

Since it was first introduced by Güth et al. (1982) the UG seems to have no rival exceeding it in popularity with experimentalists (Güth and Kocher, 2013). Equilibrium theory failed massively to predict player choices in standard versions of the game based on utility maximization models, leading to its being lauded as the death blow to simplistic theories based on individual self-interest:

The ultimatum game could hardly be simpler. If Responders maximize their own monetary payoffs, they should accept any offer. If Proposers also maximize and expect Responders to maximize, they should offer the smallest amount...The data falsify the assumption that players maximize their own profits as clearly as experimental data can...(and) is a crisp way to measure social preferences rather than a deep test of strategic thinking... (Camerer, 2003, p 43).

Not only ultimatum games, but also trust games, yield results that are commonly interpreted as a rejection of the assumption that agents are strictly self-interested. Literally, however, what we observe in ultimatum and trust games is support for other regarding choices; but it does not follow from this observation that choice is only attributable to other regarding utility (social preferences). In the above quotation, Camerer's interpretation of the data has imposed the unexamined assumption that other regarding behavior occurs if and only if preferences are other regarding. ${ }^{3}$ Suppose, however, that there

\footnotetext{
${ }^{3}$ This interpretation is common because economists do not consider non-utilitarian models of choice. For example in Fehr and Fischbacher (2002, p C1): "A substantial number of people exhibit social preferences, which means they are not solely motivated by material self-interest but also care positively or negatively for the material payoffs of relevant reference agents."
} 
exists a model of agent choice that predicts other regarding behavior in social interactions, such as twoperson ultimatum and trust games, and is based on common knowledge of strictly self-interested agents.

Adam Smith (1759) offers such a model in TMS. The short answer as to why self-interested agents exhibit other regarding behavior is that in every person's maturation, growing up in a social world, he learns to "humble the arrogance of his self-love, and bring it down to something which other men will go along with" (Smith, 1759, p 83). Smith's rich and articulate use of $18^{\text {th }}$ century English makes plain his non-utilitarian vision of human conduct driven by "fellow-feeling." To feel the approval of others when we follow a rule of conduct that resonates with others is the reinforcement that binds us to others through norms. To feel the disapproval of others when our conduct is at variance with others provokes our adaptation to correct the disharmony. Norms emerge out of these powerful socializing experiences. In TMS Smith uses "fellow-feeling" 39 times, "harmony" or "disharmony" 28 times. "Harmony" appears conjunctively in forms such as: "harmony of sentiments and affections;" "harmony and correspondence;" "harmony and contentment;" and in his explicit diminishment of a role for utilitarianism: "[T]hese affections, that harmony, this commerce, are felt...to be of more importance to happiness than all the little services that could be expected to flow from them" (Smith, 1759, p 39). Smith's concept of conduct clearly constitutes patterns that embrace all manner of useful utilitarian "little services," and it is these rule-governed patterns that defines our humanity.

Think of rules, rooted in social approval/disapproval, as mappings from specific circumstances and context into actions. "Circumstances" include payoffs because without knowing who selfinterestedly benefits or is hurt by alternative feasible actions we cannot judge the propriety of a rule. At this juncture Smith implicitly postulates common knowledge that individuals are strictly self-interested 
("self-loving"). ${ }^{4}$ Payoffs are part of the process or means of judging appropriate action, not the causal motive for action. To vary the context-action set and payoffs-is to vary the meaning of actions as signals that are conveyed by the actor, and read by others in their social responses.

Thus, in our social interactions we become accustomed to following rules that are governed by our mutual fellow-feeling with other(s). The rules we follow are conditioned by two forces that are emotional in their origin: (1) the resonating gratitude we feel when others clearly and intentionally choose actions of a beneficent tendency toward us; (2) the dissonant resentment we feel when others intentionally choose actions that are hurtful toward us. But in these judgments and reactions agents must know who benefits and who is hurt by their action. Feelings of gratitude evoke an urge to reward the beneficent actor; feelings of resentment provoke an urge to punish the hurtful actor. Because knowing that an action benefits or harms a person entails knowing that someone prefers more to less in the neighborhood of the decision, common knowledge that all are self-interested is sufficient for making these reward-benefit/punish-hurt social judgments. In other words, knowing that all are self-interested enables us to be properly other-regarding toward others. Economists and psychologists in the neoclassical tradition have neither been aware of, nor affected by, this contrary view of the role of selfinterest. In Smith and Wilson (2017, pp 10-20) we use the TMS model to interpret choice in trust games, make choice predictions in new trust games, and test those predictions. Here we extend and test the model for the UG based on a proposition in TMS that strategically qualifies Smith's primary propositions on beneficence and justice.

\section{The Motivating Proposition in Adam Smith}

We focus on the following proposition, which we state in two parts:

(A) "Beneficence is always free, it cannot be extorted by force,"

\footnotetext{
${ }^{4}$ Nowhere does Smith explicitly make this argument, but it is taken for granted in his propositions on beneficence and justice and it is easy to see that it is a sufficient condition for them.
} 
(B) "[T]he mere want of it exposes to no punishment; because the mere want of beneficence does no real positive evil...and...cannot provoke any resentment that mankind will go along with" (Smith, 1759, p 78).

In Smith and Wilson (2017) we apply (B) to test the prediction that in a trust game, if Player 1 elected not to offer to cooperate with Player 2, then Player 2, given a costly option to punish Player 1 for this "want of beneficence," would not elect to use the option. Of the 15 Player 2's, who were afforded the opportunity, not a single one invoked this punishment option! $!^{5}$

Part (A) of this proposition invites particular application to the standard form of the UG; subjects show up for an experiment, learn that they are in a UG wherein they are randomly assigned the Proposer position, and their decision is exposed without recourse to the coercive threat of rejection by the Responder. ${ }^{6}$ From the model in TMS, we get the clear implication that such conditions have predictable consequences for the actions chosen by people in these games. What is the significance of this involuntary protocol?

One perspective harks back to a paper by Ellsberg (1956), "Theory of the Reluctant Duelist," in which it was demonstrated that the minimax solution provided for zero-sum games could not be considered "general": optimal play yielded zero for each player if the players acted rationally; since any player could grantee herself zero for sure by refusing to play, such games necessarily involved reluctant

\footnotetext{
${ }^{5}$ We have since replicated this result with 8 more out of 8 Player 2's choosing to not punish want of beneficence. ${ }^{6}$ Part (B) enters in evaluating play in trust games only when we compare the frequency with which Player 1's offer to cooperate, with and without the option to punish Player $2 \mathrm{~s}$ in the event that they defect on the cooperative offer. More Player 1's offer to cooperate when the threat of punishment is an available option. But the offer to cooperate is an ambiguous signal of beneficence when coupled with Player 1 having the option to punish defection. Hence, more Players 2's defect on such offers, showing less trustworthiness-an observation consistent with Part (B); action under the extortionist threat of punishment merits a lower propensity to reward by reducing the frequency of cooperative behavior. See Smith and Wilson (2017), and Fehr and List (2004)
} 
players; hence the name of Ellsberg's classic paper. ${ }^{7}$ The UG is a constant-sum, strict opposition game

(one participant can only gain at the expense of the other), exactly as in zero-sum games. ${ }^{8}$

The other perspective, arising in the vast literature on the UG, argues that rejections of low

offers are an emotional response stemming from anger. ${ }^{9}$

\section{Equilibrium Play in Ultimatum Stage Games: Voluntary Play; Gains from Exchange}

A further indication of the reluctant duelist source of inhibitions in the standard UG is provided by an experiment in which this coercive element is replaced with a voluntary mutual interaction process that ends with a stage game identical to the UG, but with radically different outcomes.

Motivated by eBay selling procedures, Salmon and Wilson (2008) provide a game-theoretic model, and test its predictions, in which subjects engage in a two-stage process: First, a seller who has two identical units of an item, offers a single unit for purchase by multiple competing buyers in a typical

\footnotetext{
${ }^{7}$ Falk et al. (2007, p 22) examine intentions in a study reporting the results of a variation on the standard UG in which Proposers are constrained to offer only two alternative splits of 10 points (that convert into cash). The (Proposer, Responder) split that is fixed across all choices is $(8,2)$, which was always offered against one of the following single alternatives in each game $(5,5),(2,8),(8,2)$ and $(10,0)$, using the "strategy method." In one comparison there is no choice-the alternatives are identical: Proposer has no choice except to offer $(8,2)$, but $18 \%$ of them are rejected! This seems to be a "kill the messenger" signal, consistent with an aversion for playing the UG, not dissatisfaction with the Proposer's choice, who treated the Responder in the best possible way given the experimenters' imposed constraints.

${ }^{8}$ Thus in traditional game theory: "In a constant sum game, the sum of all players' payoffs is the same for any outcome. Hence, a gain for one participant is always at the expense of another...Since payoffs can always be normalized, constant sum games may be represented as (and are equivalent to) zero sum games in which the sum of all players' payoffs is always zero." See http://www.gametheory.net/dictionary/ConstantSum.html (15 August 2005).

${ }^{9}$ For the emotional perspective in experiments, see Pillutla and Murnghan (1996); O'Connor et al. (2002); Sanfey et al. (2003); Xiao and Houser (2005); Van't Wout et al. (2006). Emotion, or better, fellow-feeling, underlies Adam Smith's concepts of beneficence and injustice, arising not from a utilitarian analysis, but from our rule-following adaptation to learning from our social experience: "it is altogether absurd and unintelligible to suppose that the first perceptions of right and wrong can be derived from reason, even in those particular cases upon the experience of which the general rules are formed. These first perceptions, as well as all other experiments upon which any general rules are founded, cannot be the object of reason, but of immediate sense and feeling. It is by finding in a vast variety of instances that one tenor of conduct constantly pleases in a certain manner, and that another as constantly displeases the mind, that we form the general rules of morality. But reason cannot render any particular object either agreeable or disagreeable to the mind for its own sake" (Smith, 1759, p 320).
} 
ascending price (English) auction. The seller then makes a take-it-or-leave-it offer of her second unit to the bidder with the highest losing bid (second highest of the bid prices). If the offer is accepted the buyer makes a profit equal to the difference between his randomly drawn value and the seller's offer price. If the offer is refused, both the seller and the buyer receive zero earnings from that unit. This is an ultimatum stage game. In a treatment with two bidders, 12 of 273 offers, or $4.4 \%$ are rejected; 93 of the offers exceed the buyer's losing final bid, but only 6 are refused. The median buyer profit is only 61 cents earned on these offers. In a second treatment four buyers compete in the auction, and sellers make 111 profitable offers to their corresponding second high bid buyers; only four, or $3.6 \%$ are rejected, and the median buyer profit is 39 cents. These outcomes provide strong support for subgame perfect equilibrium in the second stage UG.

This version of the UG reflects not only voluntarism, but also gains from exchange; it is a voluntary variable-surplus ultimatum game, suggesting that we need to test not only for the effect of voluntarism, but also the combined effect of voluntarism and gains from exchange.

The variable-surplus connection is further indicated in experiments by Fouraker and Siegel (1962, p 218-9) who, two decades prior to the innovation of Güth et al (1982), studied bilateral monopoly bargaining in which, in a two-stage process, a monopoly seller quotes a take-it-or-leave it price for the sale of units to a monopsony buyer who responds with the quantity to be purchased at that price. The (Bowley) equilibrium price and quantity is for an ultimatum game with a variable surplus depending on the price and quantity chosen by the seller Proposer and the buyer Responder. Both players have complete information under a single-trial protocol that jointly determines their payoffs. Since the buyer is free to respond with a quantity choice of zero units, and is well-motivated to do so if the price is too high, this is an ultimatum game with a variable joint surplus to be divided by the monopolists. Moreover, the seller has a prominently available price at which there is a corresponding quantity that the buyer can choose that yields an equal split profit at the Pareto optimal division of the 
joint surplus. None of 10 sellers choose the equal split price; all choose prices at (or near) the equilibrium price. Seller earned profit varies asymmetrically from 2.2 to 3.6 times the buyer's profit.

\section{Voluntary Ultimatum Games for the Division of a Fixed Sum and of a Variable Sum}

Figures 1(a) and 1(b) display the extensive form games in our experiment. In Figure 1(a) Player 1, elects either to play right, yielding the minimum unit of account $(\$ 1, \$ 1)$ for each player, or to play down, volunteering to enter the fixed-sum UG as the Responder. In Figure 1(b), Player 1 elects either to play right, yielding $(\$ 1, \$ 1)$, or to play down, volunteering to enter the variable-sum UG as the Responder. Playing down by Player 1 guarantees that she will earn more than $\$ 1$ in either game as she decides whether or not to reject Player2's proposed split. We implement the signal, "voluntary play," as a choice by the Responder; we could have implemented it as a choice by the Proposer, or both could be given the opt-out alternative before the UG stage begins. We assume, as in the above auction and the bilateral monopoly experiments, that the important element is that the players have a sense of voluntary control in the ultimatum stage game. Thus, almost all retail consumer goods are sold at fixed, posted, take-it-or-leave-it prices. But only volunteer buyers walk into the vender's store or visit his internet site and there is no requirement that a buyer must buy-surely perceived by both buyer and seller as a completely voluntary process. Indeed, this is the structure of the choice sequence that we present in Figures 1(a) and (b) in distinction from the standard UG.

We recruited 48 pairs of participants to play the fixed-sum voluntary UG, and 49 to play the variable-sum version. Subjects were undergraduates at a private university with approximately 5,000 students. No student had any prior experience in an extensive or normal form game, though many had experience in at least one prior economic experiment. Participants played the extensive form game only once and in addition to their payoffs received $\$ 7$ for showing up on time. Appendix A reports the instructions for the experiment. 


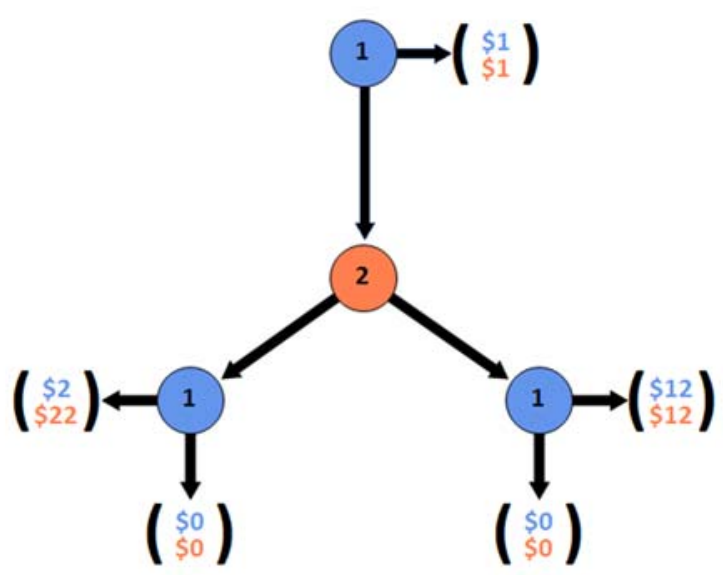

Figure 1(a). Voluntary Ultimatum Game, \$24 Fixed Sum

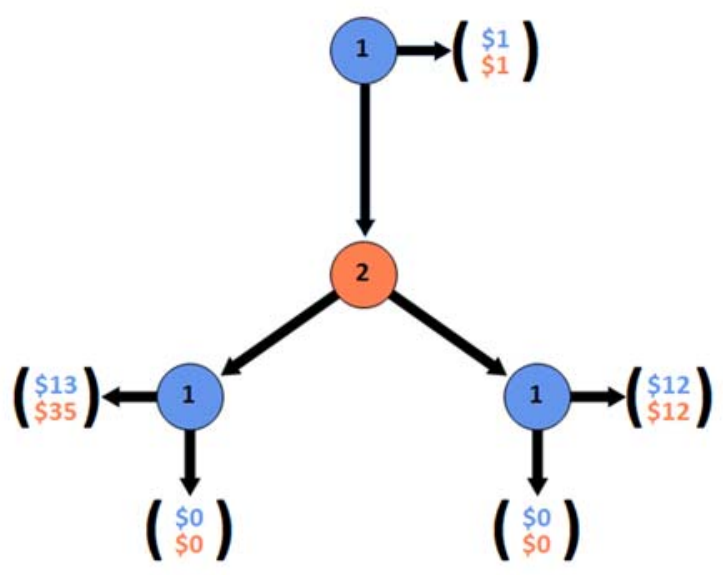

Figure 1(b). Voluntary Ultimatum Game, 2 x \$24 Variable Sum (Gains from Exchange)

Figure 2 displays the results. Notice that 6 percent in Figure 2(a) and 8 percent in Figure 2(b) volunteer not to play their respective UGs. In standard UG protocols offering choice between equal-split and a single alternative, Proposers typically do not offer splits less than (20\% for Responder, $80 \%$ for Proposer), which if offered is rejected nearly half the time (Camerer, 2003). Our alternative is more extreme: the subgame perfect equilibrium of $(\$ 2, \$ 22)$ constitutes a split of $(8 \%, 92 \%)$; and we find more subgame perfect play than has been reported in the literature for larger, more generous splits: $40 \%$ of the Proposers offer (8\%, 92\%), with $61 \%$ of Responders accepting an offer of only $8 \%$ of the pie. In comparison, Falk et al. (2003, p 24$)$ report that $31 \%$ of Proposers offer $(20 \%, 80 \%)$ against equal split, and the rejection rate is $44 \%$. Van't et al. (2006, p 566) present stand alone, single offers sequentially for rejection or acceptance. They find that all equal split offers are accepted, and offers of $10 \%$ of the fixed sum $(€ 0.5, € 4.5)$ are rejected at a rate of $80 \%$. Knight $(2012, p 11)$ reports an experiment in which the "common offer" of Proposers are presented to Responders and "...offers of...12.5\% of the pot...were rejected about $80 \%$ of the time." Finally, List and Cherry (2000) study both low stakes $(\$ 20)$ and high stakes $(\$ 400)$ repeat-play (distinct pair matching, 10 trials) ultimatum games, and report that " $27.4 \%$ (74 
out of 270 subjects) of the offers in the high stakes treatment were less than $25 \%$; of these 74 offers, $55.4 \%(41 / 74)$ were rejected" ( $p 14,17)$. In trial 10, however, unconditional rejection rates had fallen to $14.7 \%$, from their high, $44.4 \%$, on trial 2.

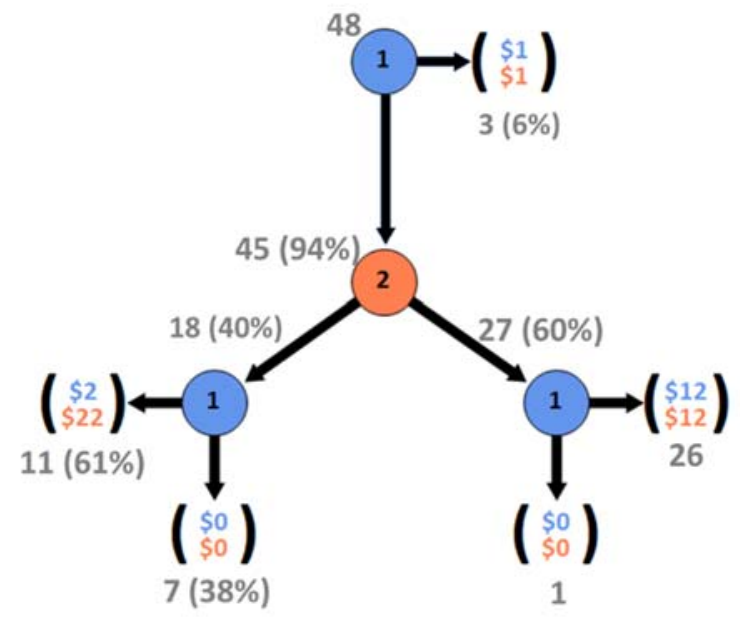

Figure 2(a). Results, Voluntary Ultimatum Game, \$24 Fixed Sum

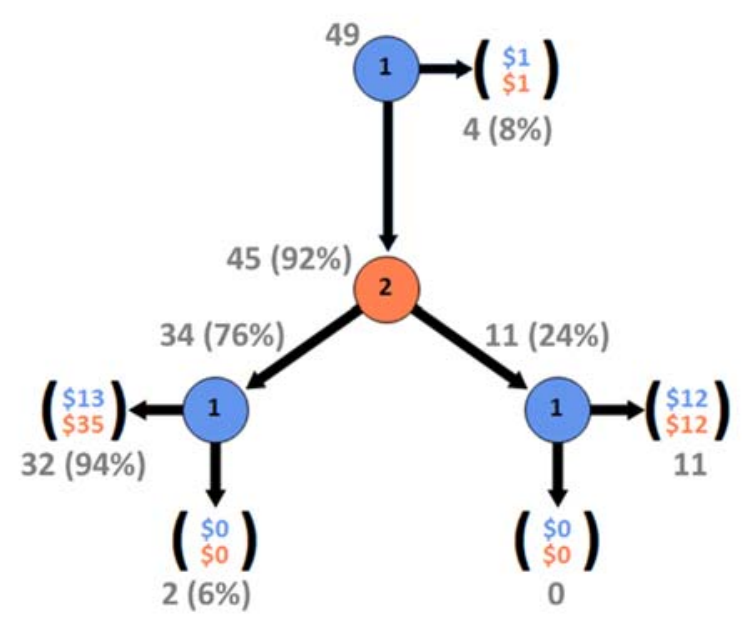

Figure 2(b). Results, Voluntary Ultimatum Game, $2 \times \$ 24$ Variable Sum

In Figure 2 (b) $76 \%$ of the Proposers offer the unequal split of $(\$ 13, \$ 35)$ or $(27 \%, 73 \%)$ and a whopping $94 \%$ of the Responders accept it. These results are entirely consistent with the high acceptance rates and low offers in Salmon and Wilson (2008). No prior experiment for involuntary, fixed sum UG division has reported such a high percentage of Proposers offering, and such a high percentage of Responders accepting, such unequal splits. Voluntarily engaging in an interaction with gains from exchange leads to high rates of subgame perfect equilibrium play with unequal outcomes.

\section{Summary and Conclusions}

In Adam Smith's model of human social conduct, actions are governed by context-dependent rules based on experience and the human capacity for mutual sympathetic fellow-feeling. The model leads to general propositions that predict action depending on the action's intended benefit or hurt, and 
thereby require common knowledge that all are self-interested; that more is beneficial, less is hurtful. In judging an action as beneficial, however, Smith $(1759, \mathrm{p} 78)$ proposes that it must be free of coercion. Applied to ultimatum games this proposition implies that the failure to observe equilibrium play is not a failure of the self-interest axiom, but rather is a consequence of the standard protocol that involves involuntary exposure to the coercive threat of veto. Accordingly, we design new constant-sum and variable-sum versions in which the first mover has an outside option which if not chosen, allows her to signal her willingness to be the responder in an ultimatum game. The results support equilibrium play at rates substantially in excess of any reported in the large UG literature.

\section{References}

Camerer, Colin F. (2003) Behavioral Game Theory. Princeton, NJ: Princeton University Press.

Ellsberg, Daniel (1956) “Theory of the Reluctant Duelist," American Economic Review, 46(5): 909-923.

Falk, Armin, Ernst Fehr and Urs Fischbacherv(2007) "On the Nature of Fair Behavior," Economic Inquiry, 41(1): 20-26.

Fehr, Ernst and Urs Fischbacher (2002) "Why Social Preferences Matter - The Impact of Non-Selfish Motives on Competition, Cooperation and Incentives," The Economic Journal 112(478):C1-C33.

Fehr, Ernst and John List. (2004) "The Hidden Costs and Returns of Incentives-Trust and Trustworthiness among CEOs," Journal of the European Economic Association, 2: 743-771.

Fouraker, Lawrence and Sidney Siegel (1962) Bargaining Behavior. New York: McGrawHill.

Güth, Werner and Martin G. Kocher (2014) "More than thirty years of ultimatum bargaining experiments: Motives, variations, and a survey of the recent literature," Journal of Economic Behavior and Organization, 108: 396-409.

Güth, Werner, Rolf Schmittberger, and Bernd Schwarze (1982) “An Experimental Analysis of Ultimatum Bargaining," Journal of Economic Behavior and Organization, 3(4): 367-388.

Knight, Simon (2012) "Fairness or anger in Ultimatum Game rejections?” Journal of European Psychology, Students, 3: 2-14. 
List, John A. and Todd L. Cherry (2000) "Learning to Accept in Ultimatum Games: Evidence from an Experimental Design that Generates Low Offers," Experimental Economics, 3: 11-29.

O'Connor, K., De Dreu, C., Schroth, H., Barry, B., Lituchy, T. and Bazerman, M. (2002) "What We Want to Do Versus What We Think We Should Do: An Empirical Investigation of Intrapersonal Conflict. Journal of Behavioral Decision Making, 15(5): 403-418.

Oechssler, Joerg, Andreas Roider, and Patrick W. Schmitz (2008) “Cooling-Off in Negotiations - Does It Work?" Working paper No. 463, University of Heidelberg, Department of Economics, April.

Ortmann, Andreas and Stephen Meardon (1995) "A game-theoretic re-evaluation of Adam Smith's Theory of Moral Sentiments and Wealth of Nations." The Classical Tradition in Economic Thought, Edited by Ingrid Hahne Rima, pp 43-61.

Pillutla, M. \& Murnighan, J. (1996) Unfairness, Anger, and Spite: Emotional Rejections of Ultimatum Offers," Organizational Behavior and Human Decision Processes, 68(3): 208-224.

Salmon, Timothy C. and Bart J. Wilson (2008) "Second Chance Offers Versus Sequential Auctions: Theory and Behavior," Economic Theory, 34: 47-67.

Smith, Adam. 1759. Theory of Moral Sentiments. Liberty Fund: Indianapolis, IN. (1982).

Smith, Vernon L. and Bart J. Wilson (2017) "Sentiments, Conduct, and Trust in the Laboratory," Social Philosophy and Policy, 34(1): 25-55.

Van't Wout, M., Kahn, R., Sanfey, A. \& Aleman, A. (2006) “Affective state and decision-making in the Ultimatum Game." Experimental Brain Research, 169(4): 564-568. 


\section{Appendix A. Experiment Instructions}

Please note that prior to making a decision, the colored arrows at the active decision node were flashing, alternating between the choices.

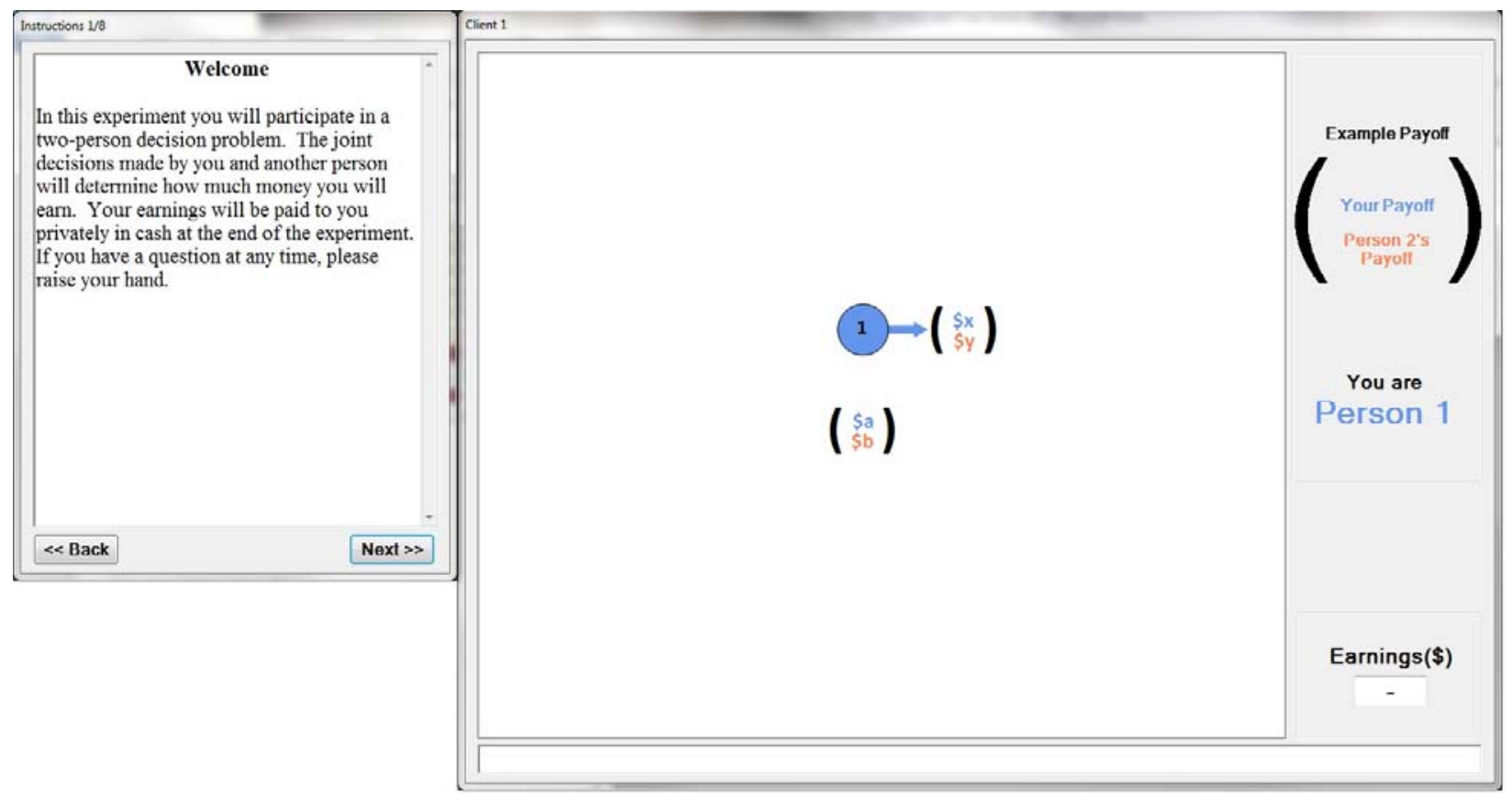

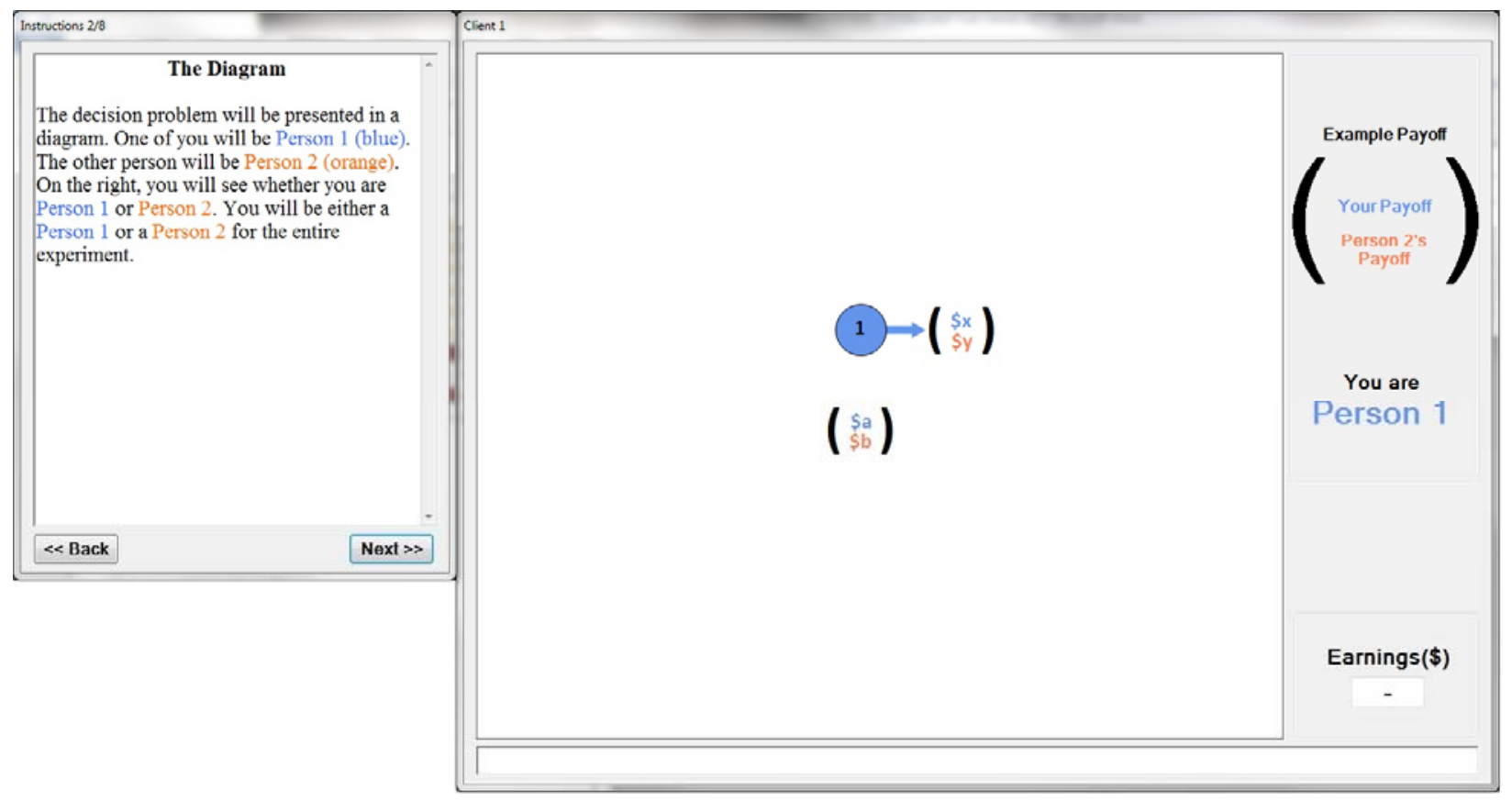




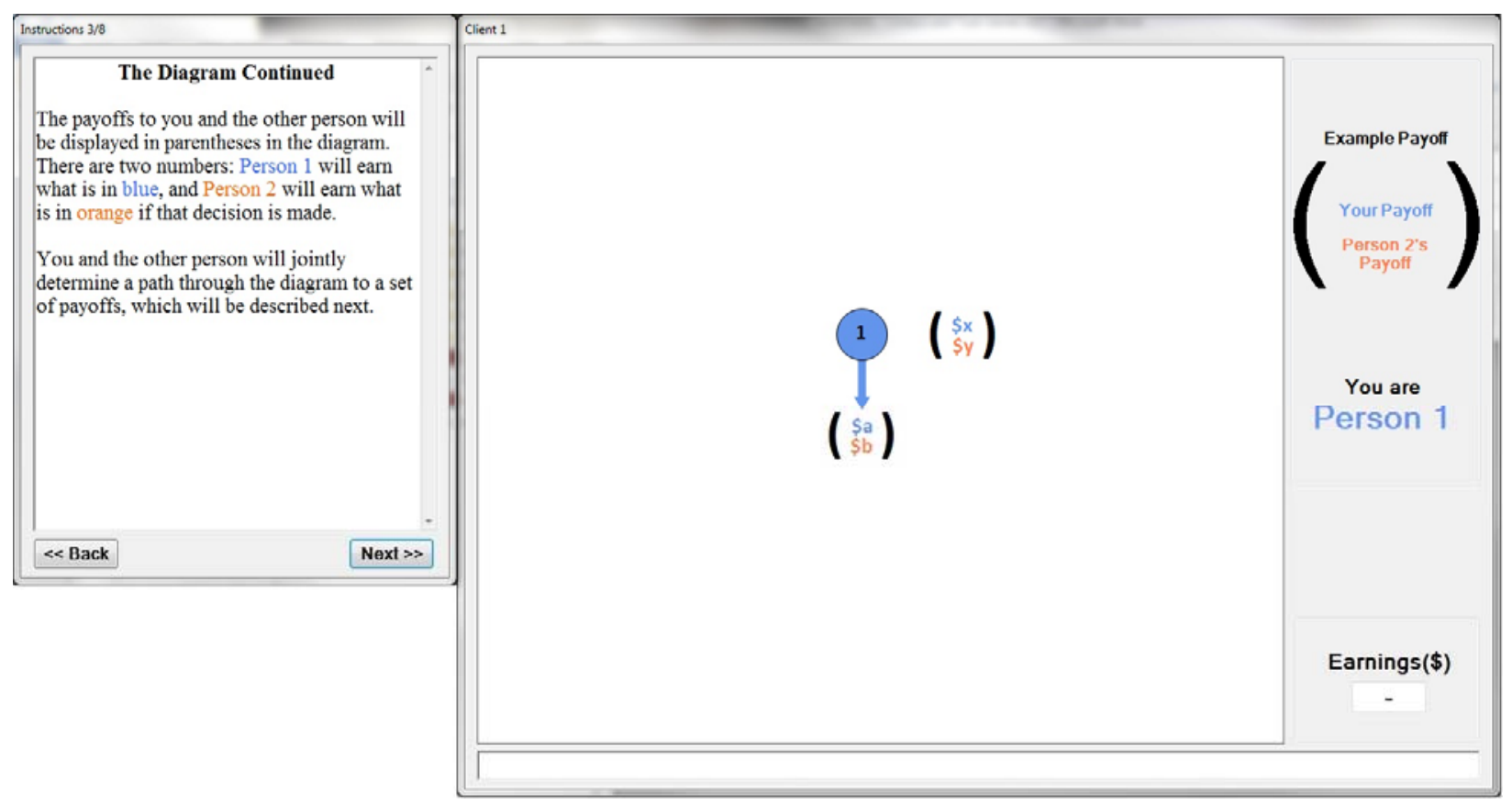

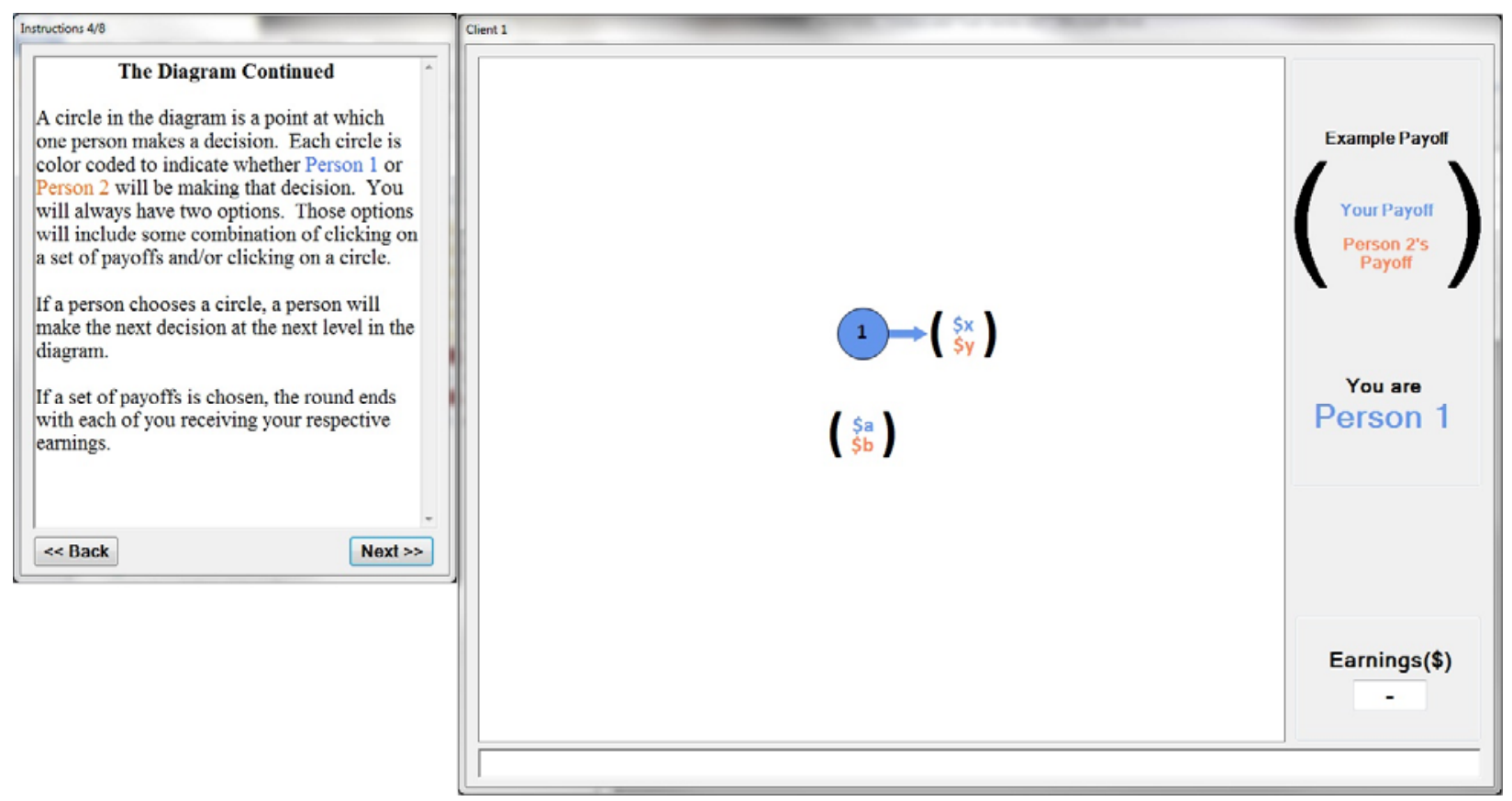




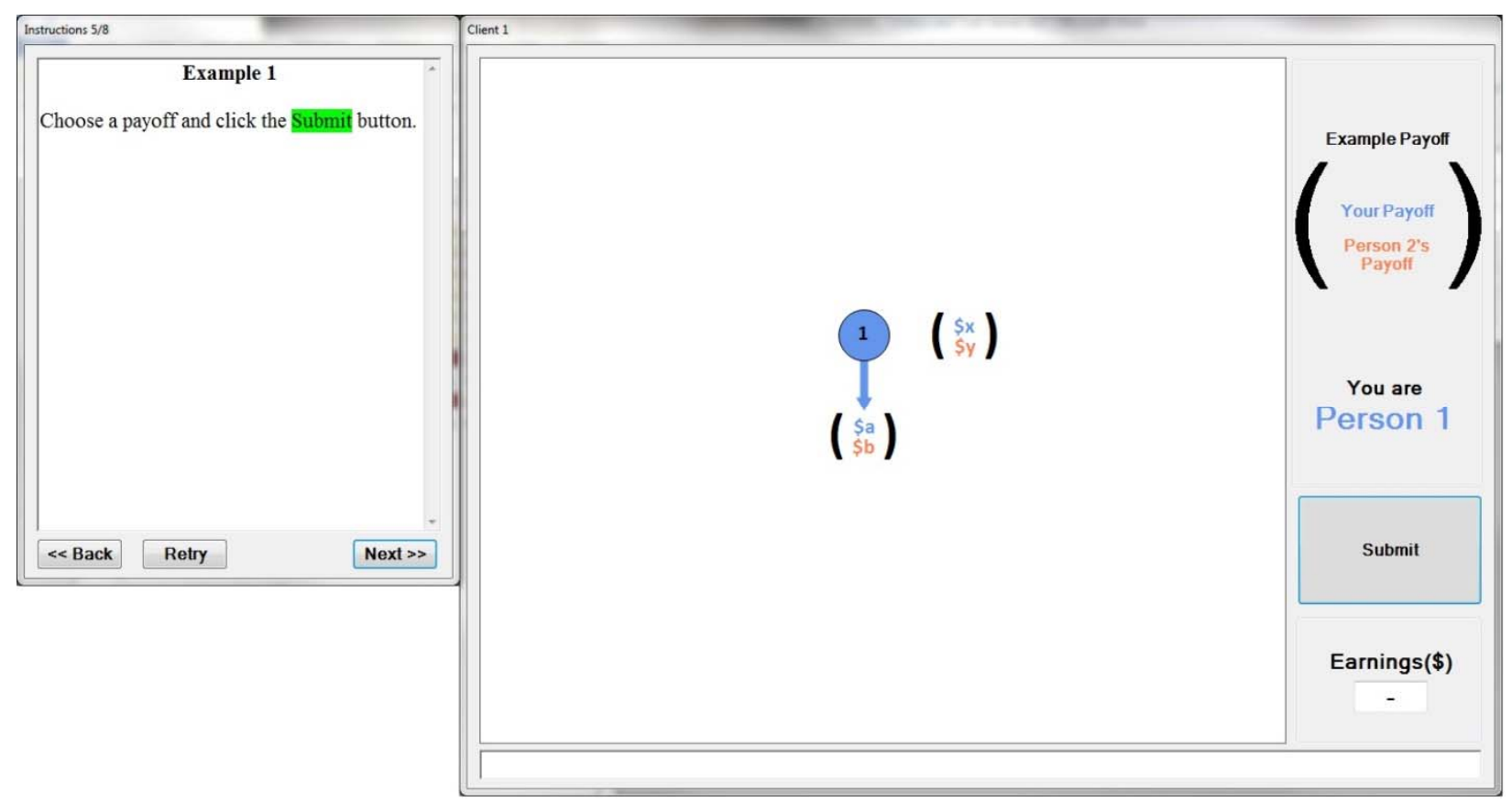

Please note that prior to making a decision, a Submit button flashed green until it was clicked.

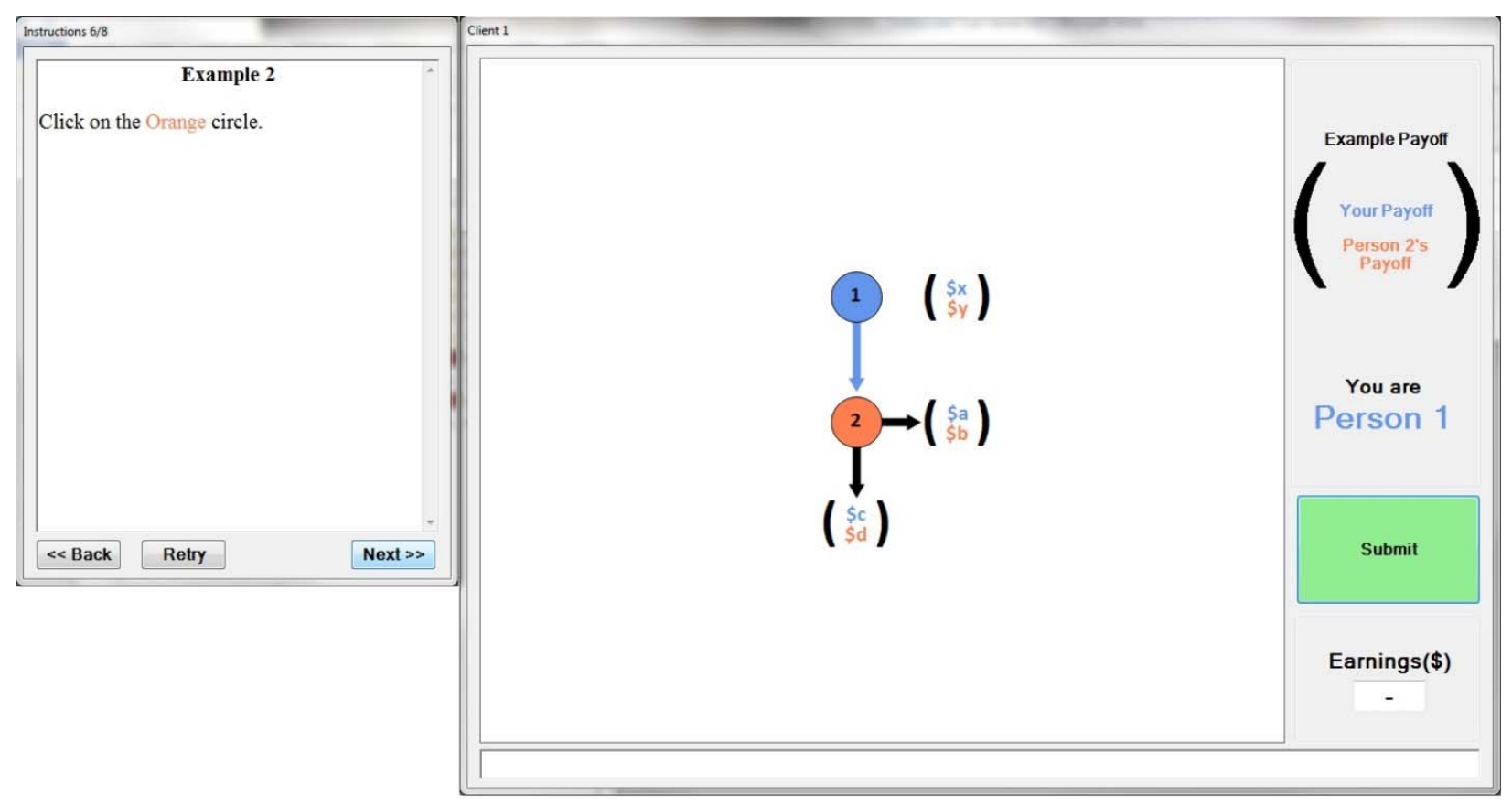



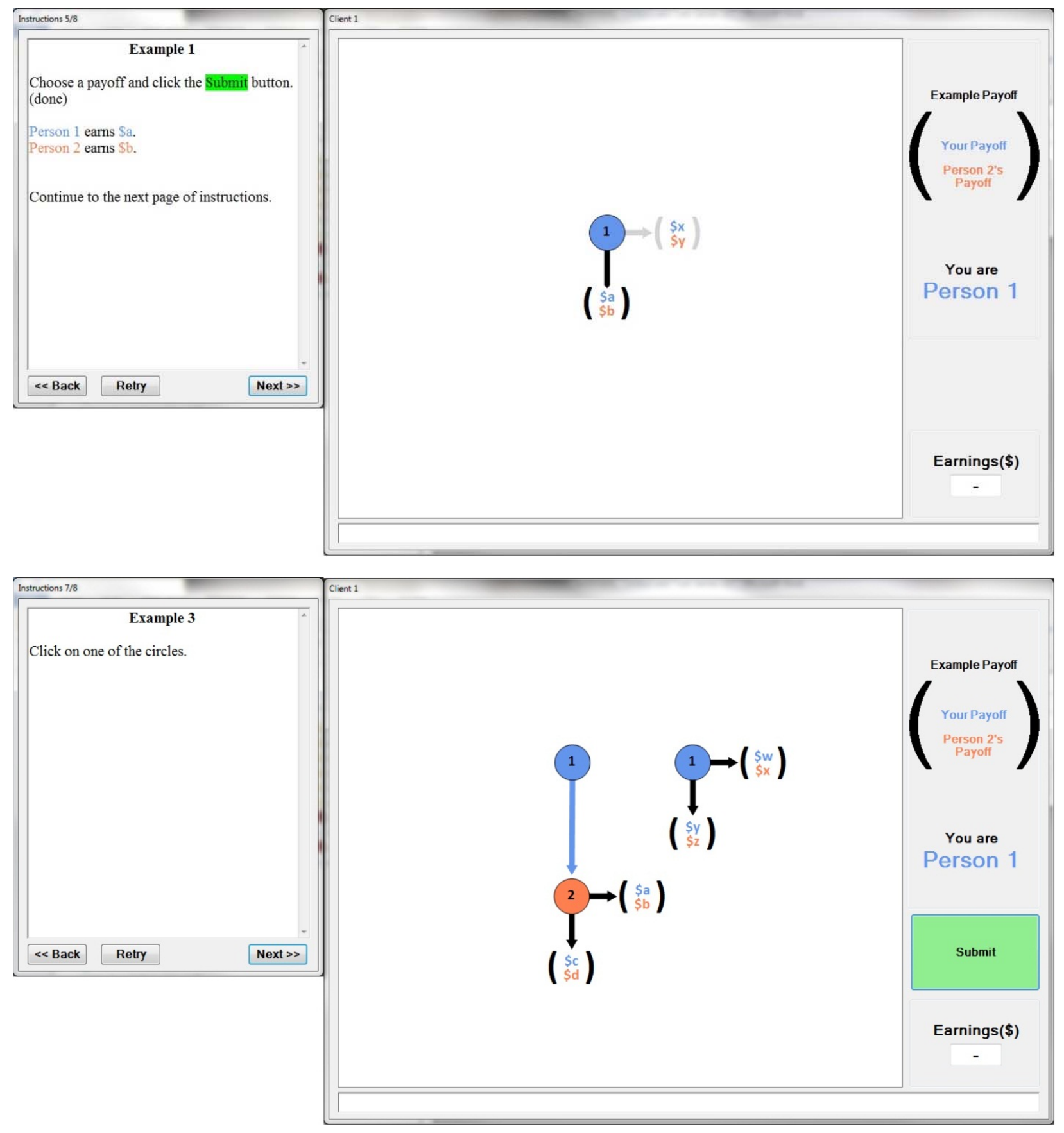

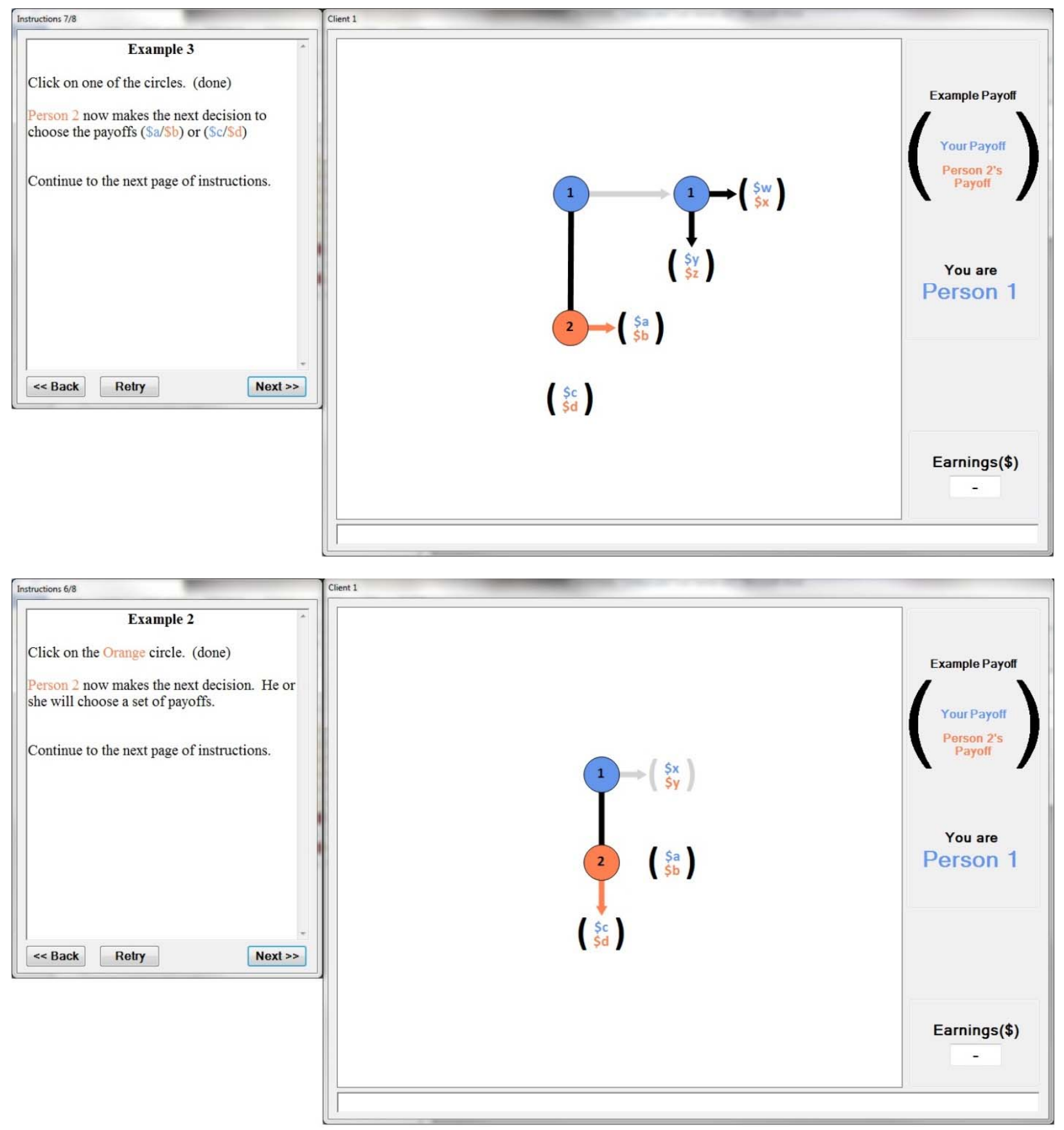


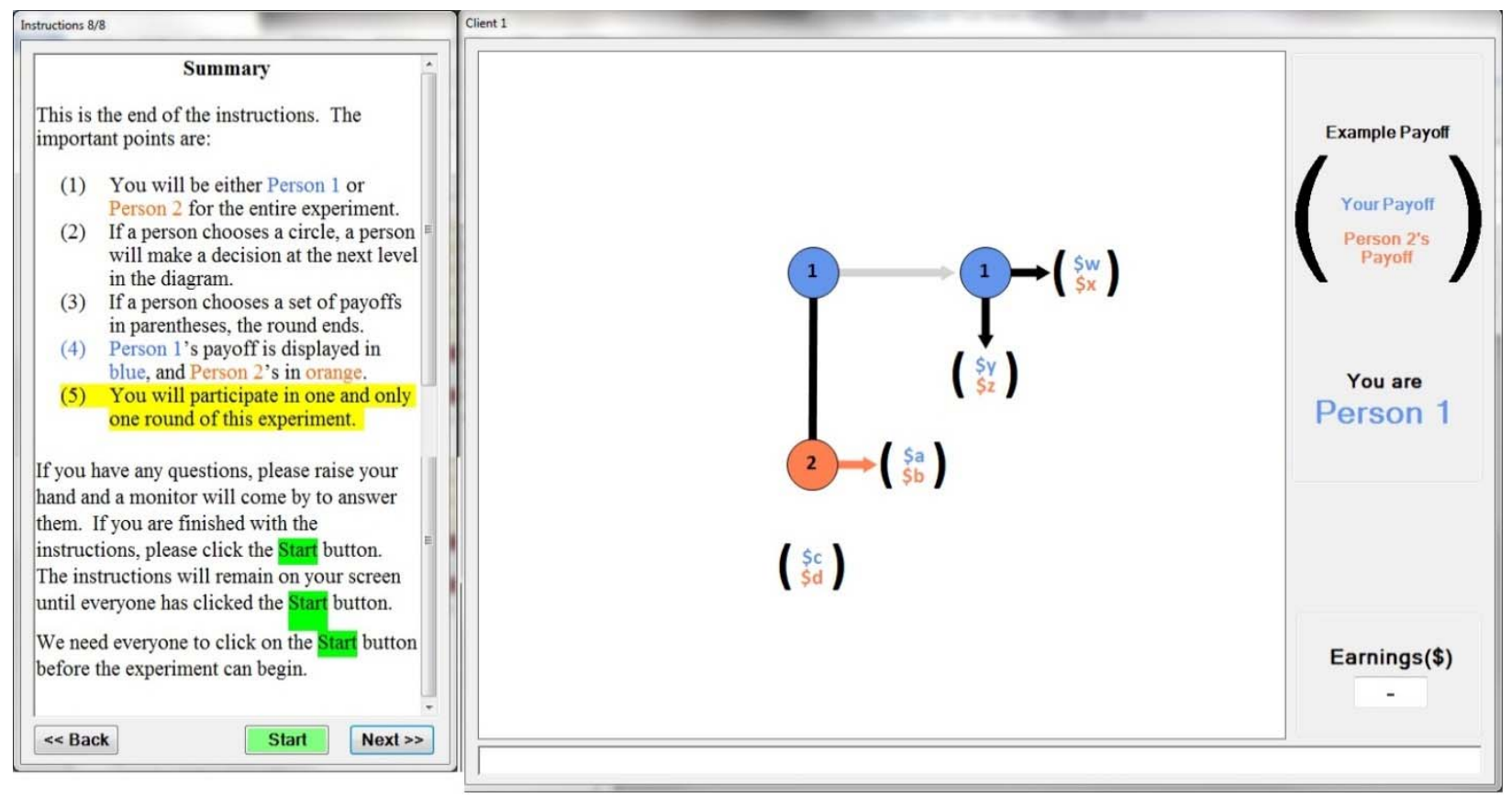

